\title{
German Adaptation of Index of Personal Reactions (G-IPR)
}

\author{
David J. Grüning \\ Heidelberg University \\ (Corresponding author) \\ Lucia L.-A. Boileau \\ University of Mannheim \\ Johanna Stolch \\ University of Mannheim
}

\section{Contacts}

If you have any questions or comments on the German IPR (G-IPR), or would like to collaborate with us on research regarding Need for Power, please feel free to contact us:

- David J. Grüning (david.gruening@stud.uni-heidelberg.de)

- Lucia L.-A. Boileau (lboileau@mail.uni-mannheim.de)

- Johanna Stolch (jstolch@mail.uni-mannheim.de)

\section{Translation comparison}

\begin{tabular}{|c|c|c|c|}
\hline & & $\begin{array}{l}\text { Index of Personal Reactions } \\
\text { (Bennett, 1988) }\end{array}$ & $\begin{array}{l}\text { German translation of Index of } \\
\text { Personal Reactions } \\
\text { (Grüning et al., 2021) }\end{array}$ \\
\hline \multicolumn{2}{|c|}{ Instruction } & $\begin{array}{l}\text { No instruction text reported in } \\
\text { the original paper. }\end{array}$ & $\begin{array}{l}\text { Bitte geben Sie für die folgenden } \\
\text { Aussagen an, wie charakteristisch } \\
\text { diese für Sie sind. }\end{array}$ \\
\hline \multicolumn{2}{|c|}{ Scoring } & $\begin{array}{l}\text { Not at all characteristic of me }= \\
0 \text {; Very much characteristic of me } \\
=4\end{array}$ & $\begin{array}{l}\text { Gar nicht charakteristisch für mich } \\
=0 ; \text { Voll und ganz charakteristisch } \\
\text { für mich }=4\end{array}$ \\
\hline \multirow[t]{5}{*}{ Ability } & Item 1 & $\begin{array}{l}\text { People see me as a forceful, } \\
\text { powerful person. }\end{array}$ & $\begin{array}{l}\text { Andere sehen mich als eine } \\
\text { energische und mächtige Person. }\end{array}$ \\
\hline & Item 2 & I know how to impress others. & $\begin{array}{l}\text { Ich weiß, wie ich andere } \\
\text { beeindrucken kann. }\end{array}$ \\
\hline & Item 3 & $\begin{array}{l}\text { I am not good at influencing } \\
\text { others. }\end{array}$ & $\begin{array}{l}\text { Ich bin nicht gut darin, andere zu } \\
\text { beeinflussen. }\end{array}$ \\
\hline & Item 4 & $\begin{array}{l}\text { When I am in charge, people } \\
\text { jump when I say jump. }\end{array}$ & $\begin{array}{l}\text { Wenn ich das Sagen habe, tun } \\
\text { andere bedingungslos, was ich sage. }\end{array}$ \\
\hline & Item 5 & $\begin{array}{l}\text { I usually know how to get what I } \\
\text { want. }\end{array}$ & $\begin{array}{l}\text { Normalerweise weiß ich, wie ich } \\
\text { bekomme, was ich will. }\end{array}$ \\
\hline
\end{tabular}


Index of Personal Reactions

(Bennett, 1988)
German translation of Index of

Personal Reactions

(Grüning et al., 2021)
Item 6 Other people tend to seek my opinion on things.

Item 7 I know how to apply pressure on others in order to get things done.

Item 8 If the situation demands it, I can have a very strong influence on people.

Item 9 I have a natural talent for influencing people.

Item 10 I am pretty good at getting my own way in most things.

Item 11 I usually achieve in my attempts to persuade others to do the things I want.

Item 12 I am confident in my ability to persuade others to do things I want.

Need for power
Item 13 I think I would enjoy having authority over others.

Item 14 I dislike having to tell others what to do.

Item 15 I am not interested in obtaining a position of power and influence.

Item 16 I do not particularly like having power over others.

Item 17 Power for its own sake doesn't interest me.

Item 18 I would enjoy being a powerful executive or politician.
Andere Leute fragen mich oft nach meiner Meinung.

Ich weiß, wie ich Druck auf andere ausüben kann, damit Dinge erledigt werden.

Wenn die Situation es erfordert, kann ich einen sehr starken Einfluss auf Menschen ausüben.

Ich habe ein natürliches Talent dazu, Menschen zu beeinflussen.

Ich bin ziemlich gut darin, meistens meinen eigenen Willen durchzusetzen.

Ich habe normalerweise Erfolg dabei, andere zu überzeugen, das zu tun, was ich will.

Ich vertraue in meine Fähigkeit, andere zu überzeugen, das zu tun, was ich will.

Ich denke, ich würde es mögen, Autorität über andere zu haben.

Ich mag es nicht, anderen sagen zu müssen, was sie tun sollen.

Ich bin nicht daran interessiert, eine Position mit Macht und Einfluss zu bekommen.

Ich mag es nicht besonders, Macht über andere zu haben.

Macht nur um ihrer selbst willen interessiert mich nicht.

Mir würde es gefallen, eine mächtige Führungskraft oder eine mächtige Politikerin zu sein. (w) Mir würde es gefallen, eine mächtige Führungskraft oder ein mächtiger Politiker zu sein. (m) 
Index of Personal Reactions

(Bennett, 1988)
German translation of Index of

Personal Reactions

(Grüning et al., 2021)

Item 19 It makes little difference to me whether I am a leader or not.

Es macht für mich wenig

Unterschied, ob ich eine

Führungskraft bin oder nicht.

Item 20 I want to be the one who makes the decisions.

Item 21 I expect to have a good deal of power someday.

Item 22 I enjoy planning things and deciding what tasks each person should do.

Need for influence
Item 23 I would like to be able to influence the actions of others.

Item 24 I like to feel that others are affected by what I have to say.

Item 25 I really enjoy it when others agree with me or see things my way.

Item 26 I feel drawn to a career that would allow me to have an important impact on other people or groups.

Item 27 It pleases me when people follow through with my suggestions.

Item 28 I am really glad when my ideas and opinions have an impact on other people.

Item 29 I prefer to work in those situations where I have some degree of influence over the decisions that are made.

Item 30 I would like feeling that I have had an impact on people's lives.
Ich möchte diejenige sein, die die Entscheidungen trifft. (w)

Ich möchte derjenige sein, der die Entscheidungen trifft. (m)

Ich denke, dass ich eines Tages viel Macht haben werde.

Ich mag es, Dinge zu planen und zu entscheiden, wer welche Aufgaben erledigen soll.

Ich würde die Handlungen anderer gerne beeinflussen können.

Ich habe gerne das Gefühl, dass das, was ich zu sagen habe, andere beeinflusst.

Ich genieße es wirklich, wenn andere mir zustimmen oder die Dinge so sehen wie ich.

Ich will eine Karriere, die es mir erlaubt, einen wichtigen Einfluss auf andere Menschen oder Gruppen zu haben.

Es gefällt mir, wenn Menschen meinen Vorschlägen folgen.

Ich freue mich, wenn meine Ideen und Meinungen Einfluss auf andere Menschen haben.

Ich arbeite gerne in Situationen, in denen ich einen gewissen Einfluss auf die Entscheidungen habe, die getroffen werden.

Ich möchte später das Gefühl haben, dass ich einen Einfluss auf das Leben anderer hatte. 
Index of Personal Reactions

(Bennett, 1988)
German translation of Index of

Personal Reactions

(Grüning et al., 2021)
Item 31 I would like it if my ideas or actions make a difference in this world, even if I am not given credit for it.
Ich fände es gut, wenn meine Ideen oder Handlungen einen Unterschied in der Welt machen, auch wenn ich dafür keine Anerkennung bekomme.
Resistance to subordination

Item 32 I don't like it when others tell me what to do.

Item 33 I can't stand it when other people boss me around.

Item 34 I try to avoid ending up in a position where I'm someone else's subordinate.

Item 35 I take orders from people in authority quite easily.

Item 36 I don't like to give in to someone else's wishes just because they happen to be my boss.

Item 37 I do not mind being subjected to the authority of another.

Item 38 I get angry when somebody tries to use their status or position to dominate me.

Item 39 I have trouble accepting it when someone has authority over me.
Ich mag es nicht, wenn andere mir sagen, was ich zu tun habe.

Ich kann es nicht ertragen, wenn andere mich herumkommandieren.

Ich versuche zu vermeiden, in einer Position zu landen, in der ich jemandem untergeordnet bin.

Ich kann Befehle von Autoritätspersonen ziemlich leicht annehmen.

Ich gebe nicht gerne den Wünschen anderer nach, nur, weil sie mir übergeordnet sind.

Es macht mir nichts aus, der Autorität einer anderen Person unterstellt zu sein.

Ich werde wütend, wenn eine Person versucht, ihren Status oder ihre Position zu benutzen, um Macht auf mich auszuüben.

Es fällt mir schwer, zu akzeptieren, wenn jemand Autorität über mich hat.

Note. Translation method: TRAPD (Harkness, 2003; Zavala-Rojas, 2017). 


\section{Scoring instructions for the original English-version of the IPR}

(Identical procedure for the G-IPR)

The table below provides a description on the calculation of the subscales and overall IPR scale.

\begin{tabular}{|l|l|l|}
\hline $\begin{array}{l}\text { Subscale /composite } \\
\text { scores }\end{array}$ & How to calculate & Content measured \\
\hline \multicolumn{2}{|c|}{ Subscale scores } \\
\hline Ability & $\begin{array}{l}\text { Average of items 1 } \\
\text { to } 12\end{array}$ & Perceived ability to wield influence and gain power. \\
\hline Need for power & $\begin{array}{l}\text { Average of items 13 } \\
\text { to } 22\end{array}$ & Need to wield power. \\
\hline Need for influence & $\begin{array}{l}\text { Average of items 23 } \\
\text { to } 31\end{array}$ & Need to wield influence. \\
\hline Resistance to subordination & $\begin{array}{l}\text { Average of items 32 } \\
\text { to } 39\end{array}$ & $\begin{array}{l}\text { Resistance to being dominated or to being in a } \\
\text { subordinate position. }\end{array}$ \\
\hline $\begin{array}{l}\text { Need for power } \\
\text { (total scale score) }\end{array}$ & Average of all items & Overall index of interpersonal reactions. \\
\hline
\end{tabular}

\section{Descriptive Statistics (English IPR)}

Descriptive statistics (i.e., mean and standard deviation), Cronbach's alpha, and test-retest reliability coefficients for the subscales of the IPR (American sample, $N=764$ and $N=154$; Bennett, 1988) are provided in the table below.

\begin{tabular}{|c|c|c|c|c|c|c|c|c|}
\hline \multirow[b]{2}{*}{$\begin{array}{l}\text { Subscale/ } \\
\text { Composite }\end{array}$} & \multicolumn{3}{|c|}{ Females $(N=422)$} & \multirow[b]{2}{*}{$\begin{array}{l}\text { Test-retest } \\
(N=103)\end{array}$} & \multicolumn{3}{|c|}{ Males $(N=342)$} & \multirow[b]{2}{*}{$\begin{array}{c}\text { Test-retest } \\
(N=51)\end{array}$} \\
\hline & $M$ & SD & $\begin{array}{c}\text { Cronbach's } \\
\text { alpha }\end{array}$ & & $M$ & $S D$ & $\begin{array}{c}\text { Cronbach's } \\
\text { alpha }\end{array}$ & \\
\hline \multicolumn{9}{|l|}{ Subscales } \\
\hline Ability & 2.47 & .61 & .87 & .81 & 2.49 & .63 & .77 & .82 \\
\hline $\begin{array}{l}\text { Need for } \\
\text { power }\end{array}$ & 2.25 & .82 & .87 & .87 & 2.46 & .78 & .86 & .83 \\
\hline $\begin{array}{l}\text { Need for } \\
\text { influence }\end{array}$ & 3.10 & .54 & .77 & .73 & 3.08 & .54 & .88 & .60 \\
\hline $\begin{array}{l}\text { Resistance to } \\
\text { subordination }\end{array}$ & 2.18 & .69 & .76 & .75 & 2.28 & .66 & .75 & .79 \\
\hline
\end{tabular}

Note: Minimum subscore of 0 and maximum of 4 . No German normative sample for the G-IPR available, yet. 


\section{Contribution}

Communication between translation parties: DJG

Contributed to the new translation of the original: DJG, LLAB

Contributed to the back translation: JS

Drafted and wrote the manuscript: DJG

Approved the submitted version for publication: DJG, LLAB, JS

\section{References}

Bennett, J. B. (1988). Power and influence as distinct personality traits: development and validation of a psychometric measure. Journal of Research in Personality, 22, 361-394.

Harkness, J. A. (2003). Questionnaire Translation. In Cross-Cultural Survey Methods, edited by J. A. Harkness, F. van de Vijver \& P. Ph. Mohler. Hoboken, NJ: Wiley.

Zavala-Rojas, D. (2017). Report of the state of the art on tools in computational linguistics and computeraided translation for enhancing survey translation. Retrieved from https://seriss.eu/resources/ deliverables/ 
Bitte geben Sie für die folgenden Aussagen an, wie charakteristisch diese für Sie sind.

\begin{tabular}{|c|c|c|c|c|c|c|}
\hline & & $\begin{array}{l}\text { Gar nicht } \\
\text { charakteristisch } \\
\text { für mich }\end{array}$ & --- & ---- & ---- & $\begin{array}{l}\text { Voll und ganz } \\
\text { charakteristisch } \\
\text { für mich }\end{array}$ \\
\hline 1 & $\begin{array}{l}\text { Andere sehen mich als eine energische und } \\
\text { mächtige Person. }\end{array}$ & 0 & 1 & 2 & 3 & 4 \\
\hline 2 & Ich weiß, wie ich andere beeindrucken kann. & 0 & 1 & 2 & 3 & 4 \\
\hline 3 & Ich bin nicht gut darin, andere zu beeinflussen. & 0 & 1 & 2 & 3 & 4 \\
\hline 4 & $\begin{array}{l}\text { Wenn ich das Sagen habe, tun andere } \\
\text { bedingungslos, was ich sage. }\end{array}$ & 0 & 1 & 2 & 3 & 4 \\
\hline 5 & $\begin{array}{l}\text { Normalerweise weiß ich, wie ich bekomme, was } \\
\text { ich will. }\end{array}$ & 0 & 1 & 2 & 3 & 4 \\
\hline 6 & $\begin{array}{l}\text { Andere Leute fragen mich oft nach meiner } \\
\text { Meinung. }\end{array}$ & 0 & 1 & 2 & 3 & 4 \\
\hline 7 & $\begin{array}{l}\text { Ich weiß, wie ich Druck auf andere ausüben } \\
\text { kann, damit Dinge erledigt werden. }\end{array}$ & 0 & 1 & 2 & 3 & 4 \\
\hline 8 & $\begin{array}{l}\text { Wenn die Situation es erfordert, kann ich einen } \\
\text { sehr starken Einfluss auf Menschen ausüben. }\end{array}$ & 0 & 1 & 2 & 3 & 4 \\
\hline 9 & $\begin{array}{l}\text { Ich habe ein natürliches Talent dazu, Menschen } \\
\text { zu beeinflussen. }\end{array}$ & 0 & 1 & 2 & 3 & 4 \\
\hline 10 & $\begin{array}{l}\text { Ich bin ziemlich gut darin, meistens meinen } \\
\text { eigenen Willen durchzusetzen. }\end{array}$ & 0 & 1 & 2 & 3 & 4 \\
\hline 11 & $\begin{array}{l}\text { Ich habe normalerweise Erfolg dabei, andere zu } \\
\text { überzeugen, das zu tun, was ich will. }\end{array}$ & 0 & 1 & 2 & 3 & 4 \\
\hline 12 & $\begin{array}{l}\text { Ich vertraue in meine Fähigkeit, andere zu } \\
\text { überzeugen, das zu tun, was ich will. }\end{array}$ & 0 & 1 & 2 & 3 & 4 \\
\hline 13 & $\begin{array}{l}\text { Ich denke, ich würde es mögen, Autorität über } \\
\text { andere zu haben. }\end{array}$ & 0 & 1 & 2 & 3 & 4 \\
\hline 14 & $\begin{array}{l}\text { Ich mag es nicht, anderen sagen zu müssen, was } \\
\text { sie tun sollen. }\end{array}$ & 0 & 1 & 2 & 3 & 4 \\
\hline
\end{tabular}


15 Ich bin nicht daran interessiert, eine Position mit

Macht und Einfluss zu bekommen.

0

1

2

3

4

16 Ich mag es nicht besonders, Macht über andere

zu haben.

0

$\begin{array}{lll}1 & 2 & 3\end{array}$

4

Macht nur um ihrer selbst willen interessiert mich nicht.

0

123

4

Mir würde es gefallen, mächtige Führungskraft

oder eine mächtige Politikerin zu sein. (w)

18 Mir würde es gefallen, eine mächtige

0

1

23

4

Führungskraft oder ein mächtiger Politiker zu

sein. $(m)$

19 Es macht für mich wenig Unterschied, ob ich eine

Führungskraft bin oder nicht.

0

1

2

3

4

Ich möchte diejenige sein, die die

20 Entscheidungen trifft. (w)

Ich möchte derjenige sein, der die

$\begin{array}{llll}0 & 1 & 2 & 3\end{array}$

4

Entscheidungen trifft. ( $m$ )

21 Ich denke, dass ich eines Tages viel Macht haben

werde.

0

1

2

3

4

Ich mag es, Dinge zu planen und zu entscheiden,

wer welche Aufgaben erledigen soll.

0

123

4

Ich würde die Handlungen anderer gerne

beeinflussen können.

- 1

1

2

3

4

Ich habe gerne das Gefühl, dass das, was ich zu

sagen habe, andere beeinflusst.

$\begin{array}{llll}0 & 1 & 2 & 3\end{array}$

4

Ich genieße es wirklich, wenn andere mir zustimmen oder die Dinge so sehen wie ich.

0

1

1

2

3

4

Ich will eine Karriere, die es mir erlaubt, einen

26 wichtigen Einfluss auf andere Menschen oder Gruppen zu haben.

Es gefällt mir, wenn Menschen meinen

Vorschlägen folgen.

0

1

2

3

4

Ich freue mich, wenn meine Ideen und

28 Meinungen Einfluss auf andere Menschen haben.

Ich arbeite gerne in Situationen, in denen ich

29 einen gewissen Einfluss auf die Entscheidungen

habe, die getroffen werden. 
30 Ich möchte später das Gefühl haben, dass ich einen Einfluss auf das Leben anderer hatte.

0

1

2

3

4

Ich fände es gut, wenn meine Ideen oder

31

Handlungen einen Unterschied in der Welt machen, auch wenn ich dafür keine Anerkennung

0

1

2

3

4

bekomme.

Ich mag es nicht, wenn andere mir sagen, was ich zu tun habe.

Ich kann es nicht ertragen, wenn andere mich herumkommandieren.

0

1

2

3

4

Ich versuche zu vermeiden, in einer Position zu

34 landen, in der ich jemandem untergeordnet bin.

Ich kann Befehle von Autoritätspersonen ziemlich leicht annehmen.

0

0

1

2

2

3

4

Ich gebe nicht gerne den Wünschen anderer

nach, nur, weil sie mir übergeordnet sind.

0

1

2

3

4

37 Es macht mir nichts aus, der Autorität einer anderen Person unterstellt zu sein.

0

1

2

3

4

Ich werde wütend, wenn eine Person versucht,

38 ihren Status oder ihre Position zu benutzen, um Macht auf mich auszuüben.

0

1

2

3

4

Es fällt mir schwer, zu akzeptieren, wenn jemand Autorität über mich hat.
0

1

2

3

4 